\title{
Existence and stability of the solutions for systems of nonlinear fractional differential equations with deviating arguments
}

\author{
Lamine Nisse and Asma Bouaziz
}

"Correspondence:

a_bouaziz@hotmail.com

Laboratory of Applied Mathematics,

Badji Mokhtar University, B.P. 12,

Annaba, 23000, Algeria

\begin{abstract}
In this paper, we give sufficient conditions for the existence and uniqueness of the solution for a class of nonlinear fractional differential systems, with variable delays. Our analysis relies on the Banach fixed point theorem. Furthermore, we prove the uniform stability of the solution. Some examples are given to illustrate our results.
\end{abstract}

Keywords: Caputo fractional derivatives; nonlinear fractional differential equations; deviating arguments; fixed point theorem; stability

\section{Introduction}

In recent years, many research works have been interested in fractional differential equations. This is due, first, to their widespread applications in diverse fields of engineering and natural sciences, and secondly to the intensive development of the theory of fractional calculus (see [1-12]). Furthermore, fractional differential equations with delays have proven more realistic in the description of natural phenomena than those without delays. Therefore, the study of these equations has drawn much attention (see e.g., [13-17]).

El-Sayed and Gaafar [16] established sufficient conditions for the existence and uniqueness of a solution to some nonlinear Riemann-Liouville fractional differential systems with constant delays. Also, they proved the stability of the solution. Recently, this study has been extended to another class of nonlinear fractional differential equations with delay in [17].

In the current paper, motivated and inspired by the works of $[16,17]$, we treat the same questions, but with time-dependent delays. Thus, we consider a system of nonlinear fractional differential equations with variable delays of the form

$$
\begin{aligned}
& { }^{c} D^{\alpha} x_{i}(t)=\sum_{j=1}^{n} f_{i j}\left(t, x_{i}(t), x_{j}\left(t-\tau_{j}(t)\right)\right), \quad i=1,2, \ldots, n, t>0, \\
& \mathbf{x}(t)=\Phi(t), \quad t \in[-\tau, 0],
\end{aligned}
$$

where ${ }^{c} D^{\alpha}$ is the Caputo fractional derivative of order $\alpha \in(0,1), \mathbf{x}(t)=\left(x_{1}(t), \ldots, x_{n}(t)\right)^{\prime}$, where' denotes the transpose of the vector, and $f_{i j}: \mathbb{R}^{+} \times \mathbb{R}^{2} \rightarrow \mathbb{R}$ are continuous functions for $i, j=1,2, \ldots, n, \tau_{j}$ are continuous real-valued functions defined on $\mathbb{R}^{+}$, such that $\tau=$ $\max \left\{\sup _{t \in \mathbb{R}^{+}} \tau_{j}(t): j=1,2, \ldots, n\right\}>0$, and $\Phi(t)=\left(\phi_{1}(t), \ldots, \phi_{n}(t)\right)^{\prime}$ is a given vector function defined on $[-\tau, 0]$ with values in $\mathbb{R}^{n}$.

O2014 Nisse and Bouaziz; licensee Springer. This is an Open Access article distributed under the terms of the Creative Commons Attribution License (http://creativecommons.org/licenses/by/2.0), which permits unrestricted use, distribution, and reproduction in any medium, provided the original work is properly cited. 
Our purpose is to establish sufficient conditions for the existence and uniqueness of a solution to the problem (1.1)-(1.2), by applying the Banach contraction principle. Furthermore, we prove the uniform stability of the solution.

While most existing research focuses on constant delays, the considered equations (1.1) contain variable delays. Moreover, it is important to note that our results are valid even in the case where the equations are of mixed type. Namely, equations of mixed type are those that have both retarded and advanced arguments. This makes a net difference with the previous works (see Remark 3.3). Thus, the present work generalizes the results obtained in $[16,17]$.

This paper is organized as follows. In Section 2, we introduce some basic definitions and notations, which are used in the sequel of the paper. In Section 3 and Section 4, we present our main results. Finally, in Section 5, two examples are given, as applications to illustrate our results.

\section{Definitions and notations}

Let us start by giving the definition of Riemann-Liouville fractional integral, and Caputo fractional derivatives. Further details of related basic properties used in the text can be found in $[3,5,8]$.

Definition 2.1 Let $\alpha \in \mathbb{R}$. The Riemann-Liouville fractional integral operator $I^{\alpha}$ is defined on $L^{1}[0, T]$ by

$$
I^{\alpha} f(t):=\frac{1}{\Gamma(\alpha)} \int_{0}^{t}(t-s)^{\alpha-1} f(s) d s, \quad t \in[0, T]
$$

where $\Gamma(\cdot)$ is the gamma function.

For $\alpha=0$, we set $I^{0}:=I d$, the identity operator. The operator $I^{\alpha}$ has the semigroup property, that is, for $\alpha, \beta \in \mathbb{R}^{+}$and $f \in L^{1}[0, T]$, the identity

$$
I^{\alpha} I^{\beta} f(t)=I^{\alpha+\beta} f(t)
$$

holds almost everywhere on $[0, T]$. Moreover, if $f \in C[0, T]$ or $\alpha+\beta \geq 1$, then the identity holds everywhere on $[0, T]$.

If $n \in \mathbb{N}^{*}$, and $D^{n} f$ (or $f^{(n)}$ ) means the $n$th derivative of a function $f$, then we have the following definition.

Definition 2.2 Let $n=[\alpha]$, and assume $D^{n} f \in L^{1}[0, T]$. The Caputo fractional derivative of order a real number $\alpha \geq 0$ is defined by

$$
{ }^{c} D^{\alpha} f(t)=I^{n-\alpha} D^{n} f(t)=\frac{1}{\Gamma(n-\alpha)} \int_{0}^{t}(t-s)^{n-\alpha-1} f^{(n)}(s) d s, \quad t \geq 0 .
$$

\section{Existence and uniqueness}

In this section we prove the existence and uniqueness of the solution of the problem (1.1)(1.2). 
Lemma 3.1 The vector function $\mathbf{x}(t):=\left(x_{1}(t), \ldots, x_{n}(t)\right)$ is a solution of the problem (1.1)(1.2) if and only if

$$
x_{i}(t)= \begin{cases}\phi_{i}(0)+\sum_{j=1}^{n} I^{\alpha} f_{i j}\left(t, x_{i}(t), x_{j}\left(t-\tau_{j}(t)\right)\right), & t>0, \\ \phi_{i}(t), & t \in[-\tau, 0], i=1,2, \ldots, n .\end{cases}
$$

Proof For $t>0$ and $i=1,2, \ldots, n,(1.1)$ can be written as

$$
I^{1-\alpha} D x_{i}(t)=\sum_{j=1}^{n} f_{i j}\left(t, x_{i}(t), x_{j}\left(t-\tau_{j}(t)\right)\right) .
$$

Applying the operator $I^{\alpha}$ on both sides of the last equality, we obtain

$$
\begin{aligned}
& I D x_{i}(t)=\sum_{j=1}^{n} I^{\alpha} f_{i j}\left(t, x_{i}(t), x_{j}\left(t-\tau_{j}(t)\right)\right), \\
& x_{i}(t)-x_{i}(0)=\sum_{j=1}^{n} I^{\alpha} f_{i j}\left(t, x_{i}(t), x_{j}\left(t-\tau_{j}(t)\right)\right) .
\end{aligned}
$$

Then

$$
x_{i}(t)=\phi_{i}(0)+\sum_{j=1}^{n} I^{\alpha} f_{i j}\left(t, x_{i}(t), x_{j}\left(t-\tau_{j}(t)\right)\right) .
$$

Let us denote by $E$ the class of all continuous column vector-valued functions $C\left(\mathbb{R}^{+}, \mathbb{R}^{n}\right)$ equipped with the norm given by

$$
\|\mathbf{x}\|_{N}=\sum_{i=1}^{n} \sup _{t \in \mathbb{R}^{+}}\left\{e^{-N t}\left|x_{i}(t)\right|\right\}, \quad \mathbf{x} \in E,
$$

where $N \in \mathbb{R}^{+}$will be chosen later. We define the integral operator $F: E \rightarrow E$ by

$$
F x_{i}(t)= \begin{cases}\phi_{i}(0)+\sum_{j=1}^{n} I^{\alpha} f_{i j}\left(t, x_{i}(t), x_{j}\left(t-\tau_{j}(t)\right)\right), & t>0, \\ \phi_{i}(t), & t \in[-\tau, 0] .\end{cases}
$$

Theorem 3.2 Assume that the following hypotheses are satisfied:

$\left(\mathrm{H}_{1}\right)$ Let $f_{i j}: \mathbb{R}^{+} \times \mathbb{R}^{2} \rightarrow \mathbb{R}$ be a continuous function, satisfying the Lipschitz condition

$$
\left|f_{i j}\left(t, x_{i}, y_{j}\right)-f_{i j}\left(t, u_{i}, v_{j}\right)\right| \leq k_{i}\left|x_{i}-u_{i}\right|+h_{j}\left|y_{j}-v_{j}\right|,
$$

where $k_{i}, h_{j}>0, i, j=\overline{1, n}$.

$\left(\mathrm{H}_{2}\right)$ For $j=1,2, \ldots, n, \tau_{j} \in C\left(\mathbb{R}^{+}, \mathbb{R}\right)$ and

$$
\tau_{j}(t)>-\tau, \quad t>0 .
$$

$\left(\mathrm{H}_{3}\right)$ For $j=1,2, \ldots, n, \exists t_{j}>0$ such that

$$
\left\{\begin{array}{l}
\tau_{j}(t) \geq t, \quad \forall t \in\left[0, t_{j}\right] \\
\left.\tau_{j}(t)<t, \quad \forall t \in\right] t_{j},+\infty[.
\end{array}\right.
$$


$\left(\mathrm{H}_{4}\right)$

$$
n \tau^{\alpha}\left[\sum_{i=1}^{n} k_{i}+\sum_{j=1}^{n} h_{j} e\right]<1 .
$$

Then the problem (1.1)-(1.2) has a unique solution.

Proof Let $\mathbf{x}, \mathbf{y} \in E$, then for $i=1,2, \ldots, n$ and $t>0$ we have

$$
\begin{aligned}
\mid F x_{i}(t) & -F y_{i}(t) \mid \\
= & \left|\sum_{j=1}^{n} \int_{0}^{t} \frac{(t-s)^{\alpha-1}}{\Gamma(\alpha)}\left\{f_{i j}\left(s, x_{i}(s), x_{j}\left(s-\tau_{j}(s)\right)\right)-f_{i j}\left(s, y_{i}(s), y_{j}\left(s-\tau_{j}(s)\right)\right)\right\} d s\right| \\
\leq & \sum_{j=1}^{n} \int_{0}^{t} \frac{(t-s)^{\alpha-1}}{\Gamma(\alpha)}\left|f_{i j}\left(s, x_{i}(s), x_{j}\left(s-\tau_{j}(s)\right)\right)-f_{i j}\left(s, y_{i}(s), y_{j}\left(s-\tau_{j}(s)\right)\right)\right| d s \\
\leq & \sum_{j=1}^{n} k_{i} \int_{0}^{t} \frac{(t-s)^{\alpha-1}}{\Gamma(\alpha)}\left|x_{i}(s)-y_{i}(s)\right| d s \\
& +\sum_{j=1}^{n} h_{j} \int_{0}^{t_{j}} \frac{(t-s)^{\alpha-1}}{\Gamma(\alpha)}\left|\phi_{j}\left(r_{j}(s)\right)-\phi_{j}\left(r_{j}(s)\right)\right| d s \\
& +\sum_{j=1}^{n} h_{j} \int_{t_{j}}^{t} \frac{(t-s)^{\alpha-1}}{\Gamma(\alpha)}\left|x_{j}\left(r_{j}(s)\right)-y_{j}\left(r_{j}(s)\right)\right| d s \\
\leq & n k_{i} \int_{0}^{t} \frac{(t-s)^{\alpha-1}}{\Gamma(\alpha)}\left|x_{i}(s)-y_{i}(s)\right| d s \\
& +\sum_{j=1}^{n} h_{j} \int_{t_{j}}^{t} \frac{(t-s)^{\alpha-1}}{\Gamma(\alpha)}\left|x_{j}\left(r_{j}(s)\right)-y_{j}\left(r_{j}(s)\right)\right| d s
\end{aligned}
$$

where $r_{j}(s)=s-\tau_{j}(s)$, thus

$$
\begin{aligned}
e^{-N t} & \left|F x_{i}(t)-F y_{i}(t)\right| \\
\leq & n k_{i} \int_{0}^{t} \frac{(t-s)^{\alpha-1}}{\Gamma(\alpha)} e^{-N(t-s)} e^{-N s}\left|x_{i}(s)-y_{i}(s)\right| d s \\
& +\sum_{j=1}^{n} h_{j} \int_{t_{j}}^{t} \frac{(t-s)^{\alpha-1}}{\Gamma(\alpha)} e^{-N\left(t-r_{j}(s)\right)} e^{-N r_{j}(s)}\left|x_{j}\left(r_{j}(s)\right)-y_{j}\left(r_{j}(s)\right)\right| d s \\
\leq & n k_{i} \sup _{\xi \in \mathbb{R}^{+}}\left\{e^{-N \xi}\left|x_{i}(\xi)-y_{i}(\xi)\right|\right\} \int_{0}^{t} \frac{(t-s)^{\alpha-1}}{\Gamma(\alpha)} e^{-N(t-s)} d s \\
& +\sum_{j=1}^{n} h_{j} \sup _{\xi \in \mathbb{R}^{+}}\left\{e^{-N r_{j}(\xi)}\left|x_{j}\left(r_{j}(\xi)\right)-y_{j}\left(r_{j}(\xi)\right)\right|\right\} \int_{t_{j}}^{t} \frac{(t-s)^{\alpha-1}}{\Gamma(\alpha)} e^{-N\left(t-r_{j}(s)\right)} d s \\
\leq & n k_{i} \sup _{\xi \in \mathbb{R}^{+}}\left\{e^{-N \xi}\left|x_{i}(\xi)-y_{i}(\xi)\right|\right\} \frac{1}{N^{\alpha}} \int_{0}^{N t} \frac{u^{\alpha-1} e^{-u}}{\Gamma(\alpha)} d u \\
& +\sum_{j=1}^{n} h_{j} \sup _{\xi \in \mathbb{R}^{+}}\left\{e^{-N \xi}\left|x_{j}(\xi)-y_{j}(\xi)\right|\right\} \frac{1}{N^{\alpha}} \int_{0}^{N\left(t-t_{j}\right)} \frac{u^{\alpha-1}}{\Gamma(\alpha)} e^{-u} e^{-N \tau_{j}\left(t-\frac{u}{N}\right)} d u
\end{aligned}
$$




$$
\begin{aligned}
\leq & n k_{i} \sup _{\xi \in \mathbb{R}^{+}}\left\{e^{-N \xi}\left|x_{j}(\xi)-y_{j}(\xi)\right|\right\} \frac{1}{N^{\alpha}} \int_{0}^{N t} \frac{u^{\alpha-1} e^{-u}}{\Gamma(\alpha)} d u \\
& +\sum_{j=1}^{n} h_{j} \sup _{\xi \in \mathbb{R}^{+}}\left\{e^{-N \xi}\left|x_{j}(\xi)-y_{j}(\xi)\right|\right\} \frac{1}{N^{\alpha}} \int_{0}^{N\left(t-t_{j}\right)} \frac{u^{\alpha-1}}{\Gamma(\alpha)} e^{-u} e^{N \tau} d u \\
\leq & \frac{n k_{i}}{N^{\alpha}} \sup _{\xi \in \mathbb{R}^{+}}\left\{e^{-N \xi}\left|x_{j}(\xi)-y_{j}(\xi)\right|\right\}+\sum_{j=1}^{n} \frac{h_{j} e^{N \tau}}{N^{\alpha}} \sup _{\xi \in \mathbb{R}^{+}}\left\{e^{-N \xi}\left|x_{j}(\xi)-y_{j}(\xi)\right|\right\} \\
\leq & \frac{n k_{i}}{N^{\alpha}}\|\mathbf{x}-\mathbf{y}\|_{N}+\sum_{j=1}^{n} \frac{h_{j} e^{N \tau}}{N^{\alpha}}\|\mathbf{x}-\mathbf{y}\|_{N} .
\end{aligned}
$$

Therefore,

$$
\begin{aligned}
\sum_{i=1}^{n} \sup _{t \in \mathbb{R}^{+}}\left\{e^{-N t}\left|F x_{i}(t)-F y_{i}(t)\right|\right\} & \leq\left[\sum_{i=1}^{n} \frac{n k_{i}}{N^{\alpha}}+\sum_{i=1}^{n} \sum_{j=1}^{n} \frac{h_{j} e^{N \tau}}{N^{\alpha}}\right]\|\mathbf{x}-\mathbf{y}\|_{N} \\
& \leq \frac{n}{N^{\alpha}}\left[\sum_{i=1}^{n} k_{i}+\sum_{j=1}^{n} h_{j} e^{N \tau}\right]\|\mathbf{x}-\mathbf{y}\|_{N} .
\end{aligned}
$$

Let us choose $N=\frac{1}{\tau}$. So, we have

$$
\sum_{i=1}^{n} \sup _{t \in \mathbb{R}^{+}}\left\{e^{-N t}\left|F x_{i}(t)-F y_{i}(t)\right|\right\} \leq n \tau^{\alpha}\left[\sum_{i=1}^{n} k_{i}+\sum_{j=1}^{n} h_{j} e\right]\|\mathbf{x}-\mathbf{y}\|_{N} .
$$

From hypothesis $\left(\mathrm{H}_{4}\right)$ we have $n \tau^{\alpha}\left[\sum_{i=1}^{n} k_{i}+\sum_{j=1}^{n} h_{j} e\right]<1$. So, $F: E \rightarrow E$ is a contraction. Hence, it has a unique fixed point $\mathbf{x}=F \mathbf{x}$ which is precisely the unique solution of our problem (1.1)-(1.2).

Remark 3.3 Note that, if for some $j$ the delay function $\tau_{j}(t)$ takes negative values, which is possible under the assumptions $\left(\mathrm{H}_{2}\right)$ and $\left(\mathrm{H}_{3}\right)$, then (1.1) are with advanced arguments. Thus, the sign of the delay functions being arbitrary, the equations of the considered system (1.1) may contain both types of deviation of argument i.e. both delay and advance. As far as we know, there are no published studies addressing these issues for such systems of equations. However, concerning boundary value problems of fractional order, some results on the existence of solutions are obtained in [18]. But in [18], the authors considered problems involving only an advanced argument, and they do not address the question about the uniqueness (and the stability) of the solution.

\section{Stability}

In this section we study the stability of the solution of the problem (1.1)-(1.2).

Definition 4.1 The solution of the problem (1.1)-(1.2) is stable if for any $\epsilon>0$, there exists $\delta>0$ such that for any two solutions $\mathbf{x}(t)=\left(x_{1}(t), \ldots, x_{n}(t)\right)^{\prime}$ and $\tilde{\mathbf{x}}(t)=\left(\tilde{x}_{1}(t), \ldots, \tilde{x}_{n}(t)\right)^{\prime}$ with the initial condition (1.2) and $\tilde{\mathbf{x}}(t)=\widetilde{\Phi}(t)$ for $t \in[-\tau, 0]$, respectively, one has $\| \Phi-$ $\widetilde{\Phi} \| \leq \delta$, implies $\|\mathbf{x}-\tilde{\mathbf{x}}\|_{N} \leq \epsilon$, where $\|\cdot\|$ denotes the supremum norm defined by $\|\Psi\|=$ $\sum_{i=1}^{n} \max _{t \in[-\tau, 0]}\left|\psi_{i}(t)\right|$, for all bounded vector function $\Psi$ from $[-\tau, 0]$ to $\mathbb{R}^{n}$. 
Theorem 4.2 Assume that hypotheses $\left(\mathrm{H}_{1}\right)$ - $\left(\mathrm{H}_{4}\right)$ in Theorem 3.2 are satisfied, then the solution of the problem (1.1)-(1.2) is uniformly stable.

Proof Let $\mathbf{x}(t)$ and $\tilde{\mathbf{x}}(t)$ be the solutions of the system (1.1) under the conditions (1.2) and $\{\tilde{\mathbf{x}}(t)=\widetilde{\Phi}(t)$ for $t \in[-\tau, 0]\}$, respectively. Then for $t>0$, from (3.1), we have

$$
\begin{aligned}
x_{i}(t)-\tilde{x}_{i}(t)= & \phi_{i}(0)-\tilde{\phi}_{i}(0) \\
& +\sum_{j=1}^{n} I^{\alpha}\left\{f_{i j}\left(t, x_{i}(t), x_{j}\left(t-\tau_{j}(t)\right)\right)-f_{i j}\left(t, \tilde{x}_{i}(t), \tilde{x}_{j}\left(t-\tau_{j}(t)\right)\right)\right\} .
\end{aligned}
$$

Therefore,

$$
\begin{aligned}
\mid x_{i}(t) & -\tilde{x}_{i}(t) \mid \\
\leq & \left|\phi_{i}(0)-\tilde{\phi}_{i}(0)\right| \\
& +\sum_{j=1}^{n} \int_{0}^{t} \frac{(t-s)^{\alpha-1}}{\Gamma(\alpha)}\left|f_{i j}\left(s, x_{i}(s), x_{j}\left(s-\tau_{j}(s)\right)\right)-f_{i j}\left(s, \tilde{x}_{i}(s), \tilde{x}_{j}\left(s-\tau_{j}(s)\right)\right)\right| d s \\
\leq & \left|\phi_{i}(0)-\tilde{\phi}_{i}(0)\right|+\sum_{j=1}^{n} k_{i} \int_{0}^{t} \frac{(t-s)^{\alpha-1}}{\Gamma(\alpha)}\left|x_{i}(s)-\tilde{x}_{i}(s)\right| d s \\
& +\sum_{j=1}^{n} h_{j} \int_{0}^{t_{j}} \frac{(t-s)^{\alpha-1}}{\Gamma(\alpha)}\left|\phi_{j}\left(s-\tau_{j}(s)\right)-\tilde{\phi}_{j}\left(s-\tau_{j}(s)\right)\right| d s \\
& +\sum_{j=1}^{n} h_{j} \int_{t_{j}}^{t} \frac{(t-s)^{\alpha-1}}{\Gamma(\alpha)}\left|x_{j}\left(s-\tau_{j}(s)\right)-\tilde{x}_{j}\left(s-\tau_{j}(s)\right)\right| d s \\
\leq & \max _{s \in[-\tau, 0]}\left|\phi_{i}(s)-\tilde{\phi}_{i}(s)\right|+n k_{i} \int_{0}^{t} \frac{(t-s)^{\alpha-1}}{\Gamma(\alpha)}\left|x_{i}(s)-\tilde{x}_{i}(s)\right| d s \\
& +\sum_{j=1}^{n} \max _{s \in[-\tau, 0]}\left|\phi_{j}(s)-\tilde{\phi}_{j}(s)\right| h_{j} \int_{0}^{t_{j}} \frac{(t-s)^{\alpha-1}}{\Gamma(\alpha)} d s \\
& +\sum_{j=1}^{n} h_{j} \int_{t_{j}}^{t} \frac{(t-s)^{\alpha-1}}{\Gamma(\alpha)}\left|x_{j}\left(s-\tau_{j}(s)\right)-\tilde{x}_{j}\left(s-\tau_{j}(s)\right)\right| d s . \\
& \\
& \\
&
\end{aligned}
$$

Hence,

$$
\begin{aligned}
& e^{-N t}\left|x_{i}(t)-\tilde{x}_{i}(t)\right| \\
& \leq e^{-N t} \max _{s \in[-\tau, 0]}\left|\phi_{i}(s)-\tilde{\phi}_{i}(s)\right|+n k_{i} \int_{0}^{t} \frac{(t-s)^{\alpha-1}}{\Gamma(\alpha)} e^{-N(t-s)} e^{-N s}\left|x_{i}(s)-\tilde{x}_{i}(s)\right| d s \\
& \quad+e^{-N t} \sum_{j=1}^{n} \max _{s \in[-\tau, 0]}\left|\phi_{j}(s)-\tilde{\phi}_{j}(s)\right| \frac{h_{j}}{\Gamma(\alpha+1)}\left[t^{\alpha}-\left(t-t_{j}\right)^{\alpha}\right] \\
& \quad+\sum_{j=1}^{n} h_{j} \int_{t_{j}}^{t} \frac{(t-s)^{\alpha-1}}{\Gamma(\alpha)} e^{-N\left(t-r_{j}(s)\right)} e^{-N r_{j}(s)}\left|x_{j}\left(r_{j}(s)\right)-\tilde{x}_{j}\left(r_{j}(s)\right)\right| d s \\
& \leq \max _{s \in[-\tau, 0]}\left|\phi_{i}(s)-\tilde{\phi}_{i}(s)\right|+\sum_{j=1}^{n} \max _{s \in[-\tau, 0]}\left|\phi_{j}(s)-\tilde{\phi}_{j}(s)\right| \frac{h_{j} t_{j}^{\alpha}}{\Gamma(\alpha+1)}
\end{aligned}
$$




$$
\begin{aligned}
& +n k_{i} \sup _{\xi \in \mathbb{R}^{+}}\left\{e^{-N \xi}\left|x_{i}(\xi)-\tilde{x}_{i}(\xi)\right|\right\} \int_{0}^{t} \frac{(t-s)^{\alpha-1}}{\Gamma(\alpha)} e^{-N(t-s)} d s \\
& +\sum_{j=1}^{n} h_{j} \sup _{\xi \in \mathbb{R}^{+}}\left\{e^{-N r_{j}(\xi)}\left|x_{j}\left(r_{j}(\xi)\right)-\tilde{x}_{j}\left(r_{j}(\xi)\right)\right|\right\} \int_{t_{j}}^{t} \frac{(t-s)^{\alpha-1}}{\Gamma(\alpha)} e^{-N\left(t-r_{j}(s)\right)} d s \\
\leq & \max _{s \in[-\tau, 0]}\left|\phi_{i}(s)-\tilde{\phi}_{i}(s)\right|+\sum_{j=1}^{n} \max _{s \in[-\tau, 0]}\left|\phi_{j}(s)-\tilde{\phi}_{j}(s)\right| \frac{h_{j} t_{j}^{\alpha}}{\Gamma(\alpha+1)} \\
& +n k_{i} \sup _{\xi \in \mathbb{R}^{+}}\left\{e^{-N \xi}\left|x_{i}(\xi)-\tilde{x}_{i}(\xi)\right|\right\} \frac{1}{N^{\alpha}} \int_{0}^{N t} \frac{u^{\alpha-1}}{\Gamma(\alpha)} e^{-u} d u \\
& +\sum_{j=1}^{n} h_{j} \sup _{\xi \in \mathbb{R}^{+}}\left\{e^{-N \xi}\left|x_{j}(\xi)-\tilde{x}_{j}(\xi)\right|\right\} \frac{1}{N^{\alpha}} \int_{0}^{N\left(t-t_{j}\right)} \frac{u^{\alpha-1}}{\Gamma(\alpha)} e^{-u} e^{-N \tau_{j}\left(t-\frac{u}{N}\right)} d u \\
\leq & \max _{s \in[-\tau, 0]}\left|\phi_{i}(s)-\tilde{\phi}_{i}(s)\right|+\sum_{j=1}^{n}\|\Phi-\tilde{\Phi}\| \frac{h_{j} t_{j}^{\alpha}}{\Gamma(\alpha+1)} \\
& +\frac{n k_{i}}{N^{\alpha}} \sup _{\xi \in \mathbb{R}^{+}}\left\{e^{-N \xi}\left|x_{i}(\xi)-\tilde{x}_{i}(\xi)\right|\right\}+\sum_{j=1}^{n} \frac{h_{j} e^{N \tau}}{N^{\alpha}} \sup _{\xi \in \mathbb{R}^{+}}\left\{e^{-N \xi}\left|x_{j}(\xi)-\tilde{x}_{j}(\xi)\right|\right\} \\
\leq & \max _{s \in[-\tau, 0]}\left|\phi_{i}(s)-\tilde{\phi}_{i}(s)\right|+\sum_{j=1}^{n}\|\Phi-\tilde{\Phi}\| \frac{h_{j} t_{j}^{\alpha}}{\Gamma(\alpha+1)} \\
& +\frac{n k_{i}}{N^{\alpha}}\|\mathbf{x}-\tilde{\mathbf{x}}\|\left\|_{N}+\sum_{j=1}^{n} \frac{h_{j} e^{N \tau}}{N^{\alpha}}\right\| \mathbf{x}-\tilde{\mathbf{x}} \|_{N} .
\end{aligned}
$$

Then

$$
\begin{aligned}
& \sum_{i=1}^{n} \sup _{t \in \mathbb{R}^{+}}\left\{e^{-N t}\left|x_{i}(t)-\tilde{x}_{i}(t)\right|\right\} \\
& \leq \sum_{i=1}^{n} \max _{s \in[-\tau, 0]}\left|\phi_{i}(s)-\tilde{\phi}_{i}(s)\right|+\sum_{i=1}^{n} \sum_{j=1}^{n}\|\Phi-\tilde{\Phi}\| \frac{h_{j} t_{j}^{\alpha}}{\Gamma(\alpha+1)} \\
& \quad+\sum_{i=1}^{n} \frac{n k_{i}}{N^{\alpha}}\|\mathbf{x}-\tilde{\mathbf{x}}\|_{N}+\sum_{i=1}^{n} \sum_{j=1}^{n} \frac{h_{j} e^{N \tau}}{N^{\alpha}}\|\mathbf{x}-\tilde{\mathbf{x}}\|_{N} \\
& \leq\|\Phi-\widetilde{\Phi}\|+\frac{n \sum_{j=1}^{n} h_{j} t_{j}^{\alpha}}{\Gamma(\alpha+1)}\|\Phi-\tilde{\Phi}\|+\frac{n \sum_{i=1}^{n} k_{i}}{N^{\alpha}}\|\mathbf{x}-\tilde{\mathbf{x}}\|_{N}+\frac{n \sum_{j=1}^{n} h_{j} e^{N \tau}}{N^{\alpha}}\|\mathbf{x}-\tilde{\mathbf{x}}\|_{N} \\
& \leq {\left[1+\frac{n \sum_{j=1}^{n} h_{j} t_{j}^{\alpha}}{\Gamma(\alpha+1)}\right]\|\Phi-\widetilde{\Phi}\|+\frac{n\left[\sum_{i=1}^{n} k_{i}+\sum_{j=1}^{n} h_{j} e^{N \tau}\right]}{N^{\alpha}}\|\mathbf{x}-\tilde{\mathbf{x}}\|_{N} . }
\end{aligned}
$$

It follows that

$$
\left[1-\frac{n\left[\sum_{i=1}^{n} k_{i}+\sum_{j=1}^{n} h_{j} e^{N \tau}\right]}{N^{\alpha}}\right]\|\mathbf{x}-\tilde{\mathbf{x}}\|_{N} \leq\left[1+\frac{n \sum_{j=1}^{n} h_{j} t_{j}^{\alpha}}{\Gamma(\alpha+1)}\right]\|\Phi-\widetilde{\Phi}\| .
$$

We choose $N=\frac{1}{\tau}$, and we have

$$
\left[1-n \tau^{\alpha}\left[\sum_{i=1}^{n} k_{i}+\sum_{j=1}^{n} h_{j} e\right]\right]\|\mathbf{x}-\tilde{\mathbf{x}}\|_{N} \leq\left[1+\frac{n \sum_{j=1}^{n} h_{j} t_{j}^{\alpha}}{\Gamma(\alpha+1)}\right]\|\Phi-\widetilde{\Phi}\| .
$$


Therefore, given any $\epsilon>0$, there exists

$$
\delta=\left[1-n \tau^{\alpha}\left[\sum_{i=1}^{n} k_{i}+\sum_{j=1}^{n} h_{j} e\right]\right]\left[1+\frac{n \sum_{j=1}^{n} h_{j} t_{j}^{\alpha}}{\Gamma(\alpha+1)}\right]^{-1} \epsilon>0,
$$

such that if $\|\Phi-\widetilde{\Phi}\|<\delta$, then $\|\mathbf{x}-\tilde{\mathbf{x}}\|_{N} \leq \epsilon$, which shows that the solution of the problem (1.1)-(1.2) is uniformly stable.

\section{Applications}

Example 5.1 Consider the problem

$$
\left\{\begin{array}{l}
{ }^{c} D^{\alpha} x_{1}(t)=\frac{10^{-3}}{x_{1}^{2}(t)+1}+\frac{10^{-2}}{x_{2}^{2}\left(t-\tau_{2}(t)\right)+1}, \\
{ }^{c} D^{\alpha} x_{2}(t)=\frac{10^{-1}}{x_{2}^{2}(t)-x_{2}(t)+1}+\frac{10^{-2}}{x_{3}^{2}\left(t-\tau_{3}(t)\right)-x_{3}\left(t-\tau_{3}(t)\right)+1}, \quad t>0, \\
{ }^{c} D^{\alpha} x_{3}(t)=\frac{10^{-3}}{x_{3}^{2}(t)+1}+\frac{10^{-2}}{x_{1}^{2}\left(t-\tau_{1}(t)\right)+1},
\end{array}\right.
$$

and

$$
\mathbf{x}(t)=\Phi(t), \quad t \in[-\tau, 0],
$$

where $\alpha=0.8, \tau_{j}(t)=\frac{4}{3}-\frac{1}{j+t}$ for $j=1,2,3 . \tau=\max _{t \in \mathbb{R}^{+}} \tau_{j}(t)=\frac{4}{3}$.

It is easy to see that the conditions $\left(\mathrm{H}_{1}\right)$ and $\left(\mathrm{H}_{2}\right)$ of Theorem 3.2 hold. Also, $\exists t_{j}=$ $\frac{4-3 j+\sqrt{9 j^{2}+24 j-20}}{6}$ such that

$$
\left\{\begin{array}{l}
\tau_{j}(t) \geq t, \quad \forall t \in\left[0, t_{j}\right] \\
\left.\tau_{j}(t)<t, \quad \forall t \in\right] t_{j},+\infty[
\end{array}\right.
$$

and

$$
3 \tau^{\alpha}\left[\sum_{i=1}^{3} k_{i}+\sum_{j=1}^{3} h_{j} e\right]=0.9523063402<1,
$$

where $k_{1}=k_{3}=10^{-3}, k_{2}=10^{-1} \frac{8 \sqrt{3}}{9}, h_{1}=h_{2}=10^{-2}$ and $h_{3}=10^{-2} \frac{8 \sqrt{3}}{9}$.

Hence, all hypotheses of Theorem 3.2 are fulfilled. Thus, the problem has a unique solution, and by Theorem 4.2 the solution is uniformly stable. Therefore, as a conclusion, the problem has a unique uniform stable solution.

Example 5.2 Consider the problem

$$
\left\{\begin{array}{l}
{ }^{c} D^{\alpha} x_{1}(t)=10^{-1}\left\{\sqrt{x_{1}^{2}(t)+1}+\sqrt{x_{2}^{2}\left(t-\tau_{2}(t)\right)+1}\right\}, \quad t>0, \\
{ }^{c} D^{\alpha} x_{2}(t)=10^{-2}\left\{x_{2}(t)+x_{1}\left(t-\tau_{1}(t)\right)\right\},
\end{array}\right.
$$

and

$$
\mathbf{x}(t)=\Phi(t), \quad t \in[-\tau, 0],
$$

where $\alpha=0.4, \tau_{j}(t)=\frac{5}{4}-\frac{1}{j+t}$ for $j=1,2 . \tau=\max _{t \in \mathbb{R}^{+}} \tau_{j}(t)=\frac{5}{4}$. 
It is easy to see that the conditions $\left(\mathrm{H}_{1}\right)$ and $\left(\mathrm{H}_{2}\right)$ of Theorem 3.2 hold. Also, $\exists t_{j}=$ $\frac{-4 j+\sqrt{16 j^{2}+8}}{8}$ such that

$$
\left\{\begin{array}{l}
\tau_{j}(t) \geq t, \quad \forall t \in\left[0, t_{j}\right] \\
\left.\tau_{j}(t)<t, \quad \forall t \in\right] t_{j},+\infty[
\end{array}\right.
$$

and

$$
2 \tau^{\alpha}\left[\sum_{i=1}^{2} k_{i}+\sum_{j=1}^{2} h_{j} e\right]=0.8943942329<1
$$

where $k_{1}=h_{2}=10^{-1}$ and $k_{2}=h_{1}=10^{-2}$.

Hence, all hypotheses of Theorem 3.2 are satisfied. Thus, the problem has a unique solution. Moreover, by Theorem 4.2 the solution is uniformly stable. Therefore, as a conclusion, the problem has a unique uniform stable solution.

\section{Competing interests}

The authors declare that they have no competing interests.

\section{Authors' contributions}

LN has proposed the main idea of this paper and has directed this study. LN and AB have conceived and drafted the manuscript. All authors read and approved the final manuscript.

\section{Acknowledgements}

The authors are very thankful to the anonymous referees for their valuable comments and constructive suggestions, which helped to improve the quality of the paper.

\section{Received: 6 June 2014 Accepted: 17 October 2014 Published: 27 Oct 2014}

\section{References}

1. Ahmad, B, Nieto, J: Existence of solution for non-local boundary value problems of higher-order nonlinear fractional differential equations. Abstr. Appl. Anal. 2009, Article ID 494720 (2009)

2. Benchohra, M, Hellal, M: Perturbed partial functional fractional order differential equations with infinite delay. J. Adv. Res. Dyn. Control Syst. 5(2), 1-15 (2013)

3. Kilbas, AA, Srivastava, HM, Trujillo, JJ: Theory and Applications of Fractional Differential Equations. North-Holland Mathematics Studies, vol. 204. Elsevier, Amsterdam (2006)

4. Liu, S, Jia, M, Tian, Y: Existence of positive solutions for boundary-value problems with integral boundary conditions and sign changing nonlinearities. Electron. J. Differ. Equ. 2010, 163 (2010)

5. Miller, KS, Ross, B: An Introduction to the Fractional Calculus and Fractional Differential Equation. Wiley, New York (1993)

6. Oldham, KB, Spanier, J: The Fractional Calculus. Academic Press, New York (1974)

7. Podlubny, I: Fractional Differential Equations. Academic Press, San Diego (1999)

8. Samko, SG, Kilbas, AA, Marichev, OI: Fractional Integral and Derivatives: Theory and Applications. Gordon \& Breach, New York (1993)

9. Tarasov, VE: Fractional Dynamics: Applications of Fractional Calculus to Dynamics of Particles, Fields and Media. Springer, Berlin (2010)

10. Tenreiro Machado, JA, Kiryakova, V, Mainardi, F: Recent history of fractional calculus. Commun. Nonlinear Sci. Numer. Simul. 16(3), 1140-1153 (2011)

11. Wang, G, Liu, W: Existence results for a coupled system of nonlinear fractional $2 \mathrm{~m}$-point boundary value problems at resonance. Adv. Differ. Equ. (2011). doi:10.1186/1687-1847-2011-44

12. Zhong, C, Fan, X, Chen, W: Nonlinear Functional Analysis and Its Application. Lanzhou University Press, Lanzhou (1998)

13. Ravichandran, C, Baleanu, D: Existence results for fractional neutral functional integro-differential evolution equations with infinite delay in Banach spaces. Adv. Differ. Equ. (2013). doi:10.1186/1687-1847-2013-215

14. Li, F: Mild solutions for abstract fractional differential equations with almost sectorial operators and infinite delay. Adv. Differ. Equ. (2013). doi:10.1186/1687-1847-2013-327

15. Bolat, Y: On the oscillation of fractional-order delay differential equations with constant coefficients. Commun. Nonlinear Sci. Numer. Simul. 19(11), 3988-3993 (2014)

16. El-Sayed, AMA, Gaafar, FM: Stability of a nonlinear non-autonomous fractional order systems with different delays and non-local conditions. Adv. Differ. Equ. (2011). doi:10.1186/1687-1847-2011-47 
17. Gao, Z, Yang, L, Luo, Z: Stability of the solutions for nonlinear fractional differential equations with delays and integral boundary conditions. Adv. Differ. Equ. (2013). doi:10.1186/1687-1847-2013-43

18. Wang, G, Ntouyas, SK, Zhang, L: Positive solutions of the three-point boundary value problem for fractional-order differential equations with an advanced argument. Adv. Differ. Equ. (2011). doi:10.1186/1687-1847-2013-43

10.1186/1687-1847-2014-275

Cite this article as: Nisse and Bouaziz: Existence and stability of the solutions for systems of nonlinear fractional

differential equations with deviating arguments. Advances in Difference Equations 2014, 2014:275

Submit your manuscript to a SpringerOpen ${ }^{\circ}$ journal and benefit from:

- Convenient online submission

- Rigorous peer review

- Immediate publication on acceptance

Open access: articles freely available online

- High visibility within the field

- Retaining the copyright to your article 